\title{
Practice and training in radiology in response to the COVID-19 pandemic: a reflection from an Irish academic tertiary referral centre
}

\author{
Colin McQuade $^{1}$ [D $\cdot$ Kathryn Hunter $^{1} \cdot$ Darragh Halpenny $^{1} \cdot$ William C. Torreggiani $^{1}$
}

Received: 15 February 2021 / Accepted: 26 February 2021 / Published online: 8 March 2021

(c) Royal Academy of Medicine in Ireland 2021

Sir,

It goes without saying that the last year has presented great challenges in the continued provision of health care. With the advent of vaccine approval and roll-out for COVID-19, we reflect upon the provision of radiology services and the impact on training in radiology over the last year.

Improved access to and significantly increased usage of online meeting platforms has been a ubiquitous experience for healthcare workers. Save for some initial technical difficulties with their roll-out, it has been our experience that remote participation in multidisciplinary meetings has been overall well received. It has proven to be particularly beneficial when reliant upon input from colleagues working across multiple sites, where previously a significant amount of time was lost travelling between sites.

The majority of Irish hospitals have been faced with the difficulty of having to cancel or curtail the provision of many outpatient and day-case services. With recurrent curtailment and reduction of outpatient activity, there has been concern among trainees and trainers alike about the potential for negative patient outcomes, as well as a potentially negative impact upon training. We, along with many other hospitals, have developed an increasing dependence on outsourcing and insourcing initiatives.

We witnessed a shift in the spectrum of acute pathology encountered in day-to-day practice. For example, in the first wave of COVID-19, intercollegiate general surgery guidance advocated for non-operative management for patients with early appendicitis.

Whilst our typical experience in recent years has been predominantly that of imaging early acute appendicitis, our focus was redirected during the first wave at the imaging and follow-up of complicated appendicitis, for example with perforation or peri-appendiceal abscess formation, of which some cases required image-guided drainage. As trainees, we had not typically encountered such cases in practice to date and so have enhanced our diagnostic skillset in some areas of practice. Due to our location, our hospital traditionally had a history of receiving trauma patients following highvelocity road traffic accidents, often associated with alcohol intake. However, with persistent closure of public houses and restaurants, we have witnessed a relative drop-off in the number of studies being performed for such indications.

With changes in day-to-day practice, it is arguable that protection of trainee teaching is now more important than ever. As a specialty, we find ourselves in the fortunate position that the bulk of our workload is digitally based. On this basis alone, training at local, national and international levels has been able to proceed. In our department, we have adapted by moving to provide tutorials using online meeting platforms. Trainees in radiology, like those from many other specialties, have a relative dependence on travelling nationally and internationally for additional training activities and exam preparatory courses. The providers of these courses have adapted, and many currently deliver these training programmes almost exclusively online. This, in turn, has led to a net financial and time benefit for some trainees, negating the need to travel.

Undoubtedly, each specialty will reflect upon its own hardships and difficulties encountered as a result of COVID-19. But, in some time, we may be able to reflect upon this period and consider that COVID-19 has acted as a catalyst for some positive change.

Colin McQuade

colin.mc.quade@icloud.com

1 Department of Radiology, Tallaght University Hospital, Dublin, Ireland 